\title{
ACTIVIDAD INSECTICIDA Y PERSISTENCIA DE UN FORMULADO A BASE DE Beauveria bassiana PARA EL CONTROL DE Diatraea saccharalis
}

\section{INSECTICIDAL ACTIVITY AND PERSISTENCE OF A FORMULATION BASED ON Beauveria bassiana FOR Diatraea saccharalis CONTROL}

\section{Jennifer Lorena García Riaño ${ }^{1 *}$, Juliana Andrea Gómez-Valderrama², Gustavo Adolfo Araque- Echeverry $^{3}$, Diego Fernando Vasquez ${ }^{4}$ y Laura Fernanda Villamizar-Rivero ${ }^{5}$}

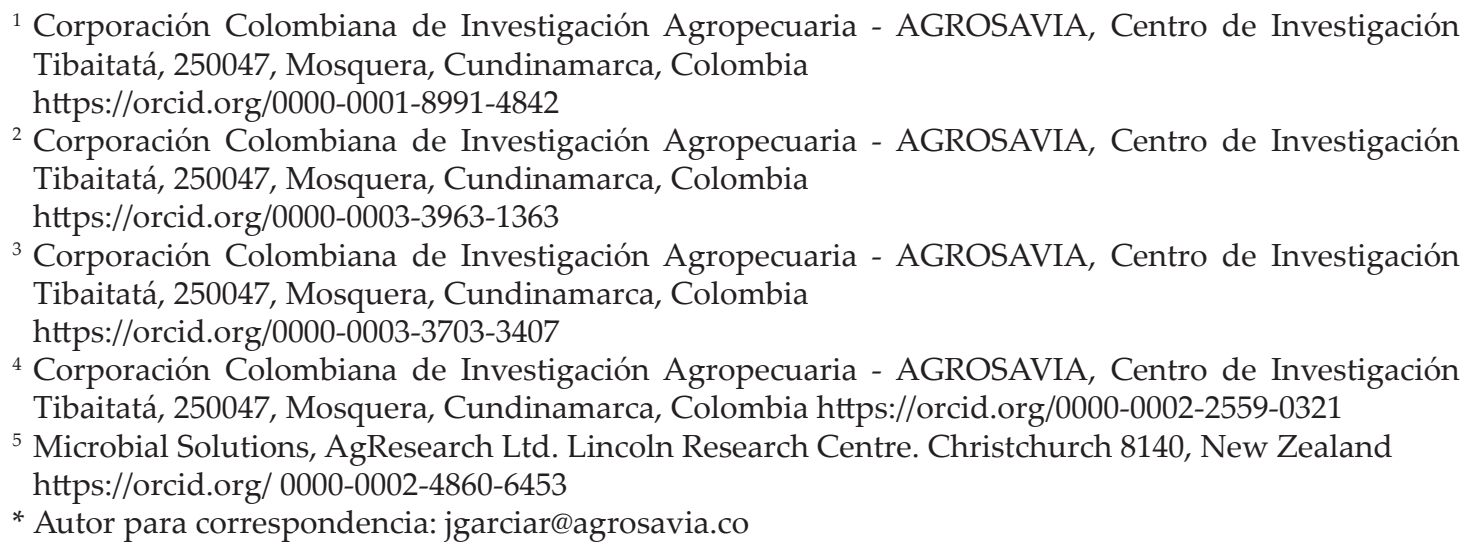

\section{RESUMEN}

El desarrollo y uso de plaguicidas microbianos para el control de plagas agrícolas se ha intensificado en los últimos años, debido a la creciente necesidad de reducir el uso de agroquímicos de alta toxicidad. Pero para suplir esta creciente necesidad de bioplaguicidas, es necesario desarrollar formulaciones adecuadas que estabilicen a los microorganismos frente a los factores ambientales de estrés que reducen su eficacia y persistencia de en campo. En este contexto, el objetivo del presente trabajo fue determinar la actividad insecticida y persistencia en plantas de caña de un concentrado emulsionable (CE) a base de Beauveria bassiana para el control del barrenador de la caña, Diatraea sp. La fotoestabilidad frente a la radiación UV tipo B y las concentraciones letales media y noventa del bioplaguicida también se determinaron bajo condiciones de laboratorio. El CE presentó una eficacia superior al $80 \%$ para el control de larvas de D. saccharalis bajo condiciones semicontroladas de campo. La formulación fotoestabilizó eficientemente los conidios, manteniendo un $68,9 \%$ de germinación después de nueve minutos de irradiación, mientras que los conidios sin formular perdieron completamente la capacidad de germinar. Este efecto también se observó para la actividad insecticida, ya que los conidios sin formular perdieron un $30 \%$ más de eficacia que los conidios formulados, después de nueve minutos de irradiación. La actividad insecticida y el recuento de conidios en plantas de caña de azúcar en campo disminuyeron a partir de la primera y segunda semana después de la aplicación, respectivamente. Aunque el CE a base de B. bassiana demostró una efectiva actividad insecticida contra $D$. saccharalis, es necesario realizar estudios de campo para optimizar la formulación y determinar el método y frecuencia de aplicación que permitan maximizar su desempeño.

Palabras clave: Conidios, Persistencia, Fotoestabilidad, Formulación, Complejo barrenador de caña. 


\section{ABSTRACT}

The development and use of microbial pesticides for the control of agricultural pests has intensified in recent years due to the increasing need for reductions in the use of highly toxic agrochemicals. To fill this growing need for biopesticides it is necessary to develop suitable formulations that stabilize microorganisms against environmental stress factors that reduce their efficacy and persistence in the field. In this context, the objective of the present work was to determine the insecticidal activity and persistence on sugarcane plants, of an emulsifiable concentrate (EC) containing Beauveria bassiana for the control of sugarcane borer, Diatraea sp. Photostability of the formulation under UV radiation type $B$ and the mean and ninety lethal concentrations were also determined under laboratory conditions. The EC reached more than $80 \%$ efficacy against $D$. saccharalis larvae under semi-controlled field conditions. The EC efficiently photostabilized the conidia, maintaining $68.9 \%$ germination after nine minutes of irradiation while unformulated conidia completely lost the ability to germinate. This effect was also observed for the insecticidal activity, with unformulated conidia losing $30 \%$ more efficacy than formulated conidia after nine minutes of irradiation. The insecticidal activity and conidia count on sugarcane plants under field conditions decreased one and two weeks post application, respectively. Although the EC containing on B. bassiana demonstrated effective insecticidal activity against $D$. saccharalis, it is necessary to carry out field trials to optimize the formulation and determine the method and frequency of application to maximize its performance.

\section{Keywords: Conidia, Persistence, Photostability, Formulation, Stem-Borer complex.}

\section{INTRODUCCION}

El complejo de especies de barrenadores de la caña de azúcar del género Diatraea spp. (Lepidoptera:Crambidae) constituye una de las principales problemáticas del cultivo a nivel mundial. El daño es ocasionado durante su estadío larval, en el cual los individuos se alimentan dentro de los tallos y construyen galerías donde viven todo su ciclo larval. El daño causado afecta el peso de los tallos y puede producir la muerte de los mismos, causando pérdidas tanto en campo como en la producción de azúcar y panela a nivel industrial, debido a la reducción del contenido de azúcares (Vargas et al., 2015)

Su manejo se ha basado en el control biológico, principalmente mediante la liberación de moscas taquínidas (Diptera: Tachinidae), que parasitan las larvas del barrenador y disminuyen las poblaciones de la plaga. Hasta hace unos años, esta estrategia era la única medida de control del insecto y no ameritaba la implementación de otras prácticas. Sin embargo, este panorama se ha modificado debido a los cambios en la distribución de especies predominantes de Diatraea sp. en las diferentes regiones, lo cual ha conllevado a niveles más bajos de parasitismo y en consecuencia al aumento del daño y las pérdidas causadas por la plaga (Vargas et al., 2013). En este sentido, recientemente se confirmó la presencia de resistencia en dos especies de Diatraea $(D$. busckella y D. tabernella) al parasitismo por parte de las moscas Lydella minense y Billaea claripalpis (Diptera: Tachinidae) (Aya et al., 2019). Aunque los programas de cría pueden eventualmente proporcionar a las moscas una capacidad mejorada para parasitar diferentes especies hospederas, se justifica un trabajo adicional para explorar tácticas alternativas de manejo para los barrenadores del tallo, compatibles con las prácticas ya implementadas, que puedan complementar el control actual.

Los microorganismos entomopatógenos constituyen una estrategia con alto potencial para el manejo del barrenador en sus primeros estadíos larvales, siendo el hongo Beauveria bassiana uno de los más destacados debido a su patogenicidad y virulencia, capacidad de generación de epizootias y facilidad de producción masiva en sustratos económicos con altos rendimientos (Mascarin y Jaronski, 2016). Durante el año 2016 se realizó la búsqueda de hongos entomopatógenos en larvas de Diatraea spp., colectadas en cultivos de caña de azúcar de diferentes regiones productoras en Colombia. El aislamiento de Beauveria bassiana codificado como Bv062, fue seleccionado por presentar actividad insecticida sobre larvas de segundo ínstar de las especies $D$. saccharalis, $D$. tabernella, D. busckella y D. indigenella. Además, este aislamiento aplicado a una concentración de $2 \times 10^{7}$ y $5 \times 10^{7}$ conidios $/ \mathrm{mL}$ de Bv062 produjo una mortalidad del $90 \%$ en larvas y del $81,95 \%$ en huevos de D. saccharalis (García et al., 2018). Sin embargo, la persistencia de los conidios de hongos entomopatógenos en el ambiente es usualmente baja, debido al efecto de condiciones ambientales como la luz ultravioleta, la temperatura y la precipitación (Inglis et al., 1997), las cuales se hacen más drásticas en aplicaciones foliares como es el caso del cultivo de caña. La formulación de los microorganismos entomopatógenos con adyuvantes de diferente funcionalidad, permiten 
mejorar la estabilidad del producto tanto en campo como en almacenamiento, asegurando que su eficacia sea consistente después de su aplicación (Díaz et al., 2019). Las formulaciones están compuestas por el ingrediente activo encargado de ejercer la actividad biológica y los auxiliares de formulación o excipientes, dentro de los cuales se pueden incluir sustancias que estabilizan el ingrediente activo, aseguran la permanencia sobre el insecto blanco, retienen la humedad y promueven la dispersión del bioproducto (Ravensberg, 2011; Díaz et al., 2019).

En este trabajo, los conidios de B. bassiana Bv062 fueron utilizados como principio activo de un prototipo de bioplaguicida formulado como un concentrado emulsionable (CE), el cual fue caracterizado determinando su actividad insecticida sobre larvas de $D$. saccharalis en condiciones controladas de laboratorio y en plantas de caña, y evaluando su fotoestabilidad y persistencia.

\section{METODOLOGÍA}

\section{Insecto}

Los experimentos se llevaron a cabo con larvas de $D$. saccharalis provenientes de la colonia ubicada en la Unidad de Crías de Insectos de AGROSAVIA ubicada en Mosquera, Cundinamarca (Colombia), que se estableció con larvas donadas por Cenicaña (Centro Colombiano de Investigación de la caña de azúcar). La cría fue mantenida en condiciones de temperatura de $25 \pm 5^{\circ} \mathrm{C}$ con una humedad relativa de $60 \pm 10 \%$ y un fotoperíodo de $16 \mathrm{~h}$ de luz: $8 \mathrm{~h}$ de oscuridad. Las larvas fueron alimentadas con la dieta artificial descrita por Lastra y Gómez (2006).

\section{Microorganismo y medio de cultivo}

El aislamiento de B. bassiana codificado como Bv062, fue obtenido a partir de una larva de Diatraea sp., proveniente de un cultivo de caña panelera ubicado en el Departamento de Boyacá (García et al., 2018). El hongo se encuentra conservado en el Banco de Germoplasma de la Colección de Microorganismos con Interés en Control Biológico de la Corporación Colombiana de Investigación Agropecuaria (Agrosavia) y para su activación fue cultivado en Agar Papa Dextrosa (PDA) suplementado con $0,1 \%$ de cloranfenicol e incubado durante 10 días a $25^{\circ} \mathrm{C}$. Este aislamiento fue registrado en el Registro Nacional de Colecciones (RNC 129) y fue colectado bajo el permiso de colección marco No. 1466 de 2014. La investigación se encuentra en el marco del Contrato No. 168 de 2017 para el "Acceso a Recursos Genéticos y Productos Derivados en Colombia".

\section{Producción y formulación de conidios}

La producción de conidios se realizó en fermentación semisólida. Para ello, se utilizaron bandejas de aluminio de $10,5 \mathrm{~cm} \times 17,5 \mathrm{~cm}$ que contenían como sustrato 10 gramos de arroz partido molido estéril con un tamaño de partícula entre 200 y $500 \mu \mathrm{m}$, el cual fue humedecido con $80 \mathrm{~mL}$ de agua estéril. Previo a la inoculación, se colocó una tela de muselina estéril sobre la superficie del sustrato y posteriormente se inocularon $10 \mathrm{~mL}$ de una suspensión de conidios preparada en una solución de Tween 80 al 0,1\% y ajustada a una concentración de $1 \times 10^{6}$ conidios/ mL mediante conteo en cámara de Neubauer. Las bandejas inoculadas se cubrieron con una película plástica y se incubaron a $25^{\circ} \mathrm{C} \pm 2{ }^{\circ} \mathrm{C}$ y $60 \pm 10 \%$ HR por ocho días. Pasado ese tiempo, la tela de muselina fue retirada y los conidios adheridos a ella fueron separados con ayuda de una espátula estéril y fueron secados durante 5 días a $25^{\circ} \mathrm{C}$ hasta obtener una humedad del $7 \%$. Posteriormente, los conidios secos fueron cosechados y formulados como un concentrado emulsionable (CE), suspendiéndolos en una concentración del 1\% en un vehículo oleoso compuesto por la mezcla de un aceite vegetal (45\%), aceite mineral $(45 \%)$ y una mezcla de tensioactivos (9\%). El bioplaguicida formulado fue posteriormente almacenado a $4^{\circ} \mathrm{C}$ en recipientes herméticamente cerrados hasta su uso. Inmediatamente después de la formulación, el CE presentó una concentración de $1 \times 10^{9}$ conidios/ $\mathrm{mL}$, germinación de conidios del $95 \%$, eficacia superior al $90 \%$ en condiciones de laboratorio empleando una concentración de $1 \times 10^{7}$ conidios/ $\mathrm{mL}$ y un contenido de contaminantes (hongos filamentosos, levaduras y bacterias) inferior a 1000 Unidades Formadoras de Colonia (UFC) por mL.

\section{Concentraciones letales del CE sobre larvas de D. saccharalis bajo condiciones de laboratorio}

El CE fue reconstituido en agua ajustando seis concentraciones finales: $5 \times 10^{5}, 1 \times 10^{6}, 5 \times 10^{6}, 1 \times 10^{7}$, $5 \times 10^{7}, 1 \times 10^{8}$ conidios/mL. Para el bioensayo se siguió la metodología descrita por Garcia et al. (2018). Treinta larvas de segundo ínstar de $D$. saccharalis fueron inoculadas aplicando $2 \mu \mathrm{L}$ del tratamiento en el dorso. Cada larva inoculada se dispuso en un vaso de plástico de media onza que contenía un grano de maíz; luego, se ubicaron en grupos de 10 en cajas Selloplus ${ }^{\circledR}$ de $700 \mathrm{~mL}$ (unidad experimental), para un total de tres cajas por tratamiento (triplicado). Las cajas se incubaron a $26 \pm 2^{\circ} \mathrm{C}$ y $70 \%$ de HR. Se contó con un testigo absoluto, en el cual las larvas no fueron sometidas a ningún tratamiento. La mortalidad se evaluó cada tres días hasta que todos los individuos murieron o pasaron al estado de pupa y los resultados fueron sometidos a un análisis 
Probit con el programa POLO Plus 2.0 (LeOra Software) para determinar las concentraciones letales. Las larvas muertas se ubicaron en cámara húmeda a $25^{\circ} \mathrm{C}$ durante siete días para evidenciar los signos de la infección fúngica (Tabla 1).

\section{Evaluación de dosis del bioplaguicida bajo} condiciones semicontroladas de campo

El ensayo se realizó en un cultivo experimental de caña de azúcar, ubicado en el municipio de Barbosa, departamento de Santander, Colombia (Sede Cimpa Agrosavia, 556 $50.0^{\prime \prime} \mathrm{N}$ $\left.73^{\circ} 36^{\prime} 20.5^{\prime \prime} \mathrm{W}\right)$, ubicado a $1600 \mathrm{msnm}$. El lote utilizado fue de $300 \mathrm{~m}^{2}$ sembrado con caña variedad CC93-7711 de dos meses y medio de edad. Los tratamientos consistieron en una dosis media calculada con base en la concentración letal 90 determinada en el ensayo de laboratorio $(1,44 x$ $10^{7} \mathrm{con} / \mathrm{mL}$ ), el volumen de aplicación por planta $(2 \mathrm{~mL})$ y la densidad de plantas (110.000 tallos/ ha), la cual correspondió a 3,2 $\times 10^{12}$ conidios/ha, una dosis inferior correspondiente a la mitad de la dosis media $\left(1,6 \times 10^{12}\right.$ conidios/ha y una dosis superior correspondiente al doble de la dosis media $\left(6,4 \times 10^{12}\right.$ conidios/ha). Adicionalmente, se contó con un testigo absoluto, en el cual las plantas no fueron aplicadas con el bioplaguicida.

El diseño experimental fue de bloques completos al azar (tres bloques), para un total de 12 parcelas, cada una de $25 \mathrm{~m}^{2}$. Cada parcela contó con cuatro surcos sobre los cuales se realizó la aplicación de los tratamientos empleando una fumigadora manual de 5L Royal Condor ${ }^{\circledR}$ Handy. La aplicación se realizó entre las 8 y las $10 \mathrm{am}$. Durante la ejecución del experimento, se presentó una temperatura promedio de $17,9 \pm 4^{\circ} \mathrm{C}$ y una humedad relativa del $60 \pm 10 \%$.

Aproximadamente una hora después de la aplicación se realizó la infestación con larvas de $D$. saccharalis de segundo ínstar, colocando cuidadosamente seis larvas por planta en 10 plantas de los dos surcos centrales. Siete días después del montaje del ensayo, las larvas encontradas fueron recolectadas y llevadas al laboratorio donde se ubicaron individualmente en recipientes plásticos de $15 \mathrm{~mL}$ con un grano de maíz. Se registró el número de sobrevivientes cada tres días por 12 días, y con el último dato se calculó el porcentaje de eficacia utilizando la fórmula de Abbott (Abbott, 1925):

Eficacia $(\%)=1-\frac{n \text { en el tratamiento después de aplicación }}{n \text { en Control después de aplicación }}$

En donde $n$ corresponde a la población de insectos recuperados.

Con el fin de estimar el cubrimiento de las hojas con los conidios del hongo (densidad de propágulos en un área determinada), antes de la aplicación del CE se colocaron dos laminillas cubreobjetos de $4,4 \mathrm{~cm}^{2}$ en tres plantas de cada parcela, ubicando una en el haz de una hoja localizada hacia el extremo de la planta (Posición 1) y la otra en la hoja cercana a la yagua (Posición 2). Después de la aplicación, las laminillas se recogieron y se depositaron por separado en vasos de plástico de $15 \mathrm{~mL}$, los cuales se mantuvieron bajo condiciones de refrigeración hasta el procesamiento de las muestras. Las laminillas fueron lavadas con $500 \mu \mathrm{L}$ de Tween 80 al 0,1\%, agitando durante una hora a $125 \mathrm{rpm}$. Posteriormente, se determinó la concentración de la suspensión obtenida después del lavado mediante recuento de conidios en cámara de Neubauer y se realizaron diluciones seriadas que se sembraron por duplicado en agar PDA con Cloranfenicol $0,1 \%$ y Tritón $0,1 \%$, para el recuento de unidades formadoras de colonia después de 5 días de incubación a $25^{\circ} \mathrm{C}$.

Estabilidad del bioplaguicida frente a la radiación ultravioleta tipo $B$ en condiciones de laboratorio

El efecto de la radiación ultravioleta sobre la germinación y la actividad insecticida de los conidios sin formular y formulados como CE, se evaluó bajo condiciones de laboratorio. Los tratamientos reconstituidos en agua fueron ajustados a $1 \times 10^{7} \mathrm{con} / \mathrm{mL}$ y se adicionaron 500 $\mu \mathrm{L}$ de las suspensiones de cada tratamiento en un vaso de plástico de $15 \mathrm{~mL}$, los cuales fueron expuestos a la radiación ultravioleta tipo B (UV-

Tabla 1. Concentraciones letales del CE a base de B. bassiana Bv062 sobre larvas de segundo ínstar de D. saccharalis.

Table 1. Lethal concentrations of EC based on B. bassiana Bv062 on second instar larvae of D. saccharalis

\begin{tabular}{|c|c|c|c|c|c|c|c|}
\hline \multirow{2}{*}{$\begin{array}{c}\mathrm{CL}_{50} \\
\text { (con/mL) }\end{array}$} & \multicolumn{2}{|c|}{$\begin{array}{c}\text { Límites de confianza } \\
\mathrm{CL}_{50}(\mathrm{con} / \mathrm{mL})\end{array}$} & \multirow{2}{*}{$\begin{array}{c}\mathrm{CL}_{90} \\
\text { (con/mL) }\end{array}$} & \multicolumn{2}{|c|}{$\begin{array}{l}\text { Límites de confianza } \\
\mathrm{CL}_{90}(\mathrm{con} / \mathrm{mL})\end{array}$} & \multicolumn{2}{|c|}{ Heterogeneidad } \\
\hline & Inferior & Superior & & Inferior & Superior & $\chi^{2}$ & gl \\
\hline $9,27 \times 10^{5}$ & $1,54 \times 10^{5}$ & $2,18 \times 10^{5}$ & $1,44 \times 10^{7}$ & $5,56 \times 10^{6}$ & $1,71 \times 10^{8}$ & 6,00 & 4 \\
\hline
\end{tabular}


B) emitida por una lámpara monocromática (302 nm) (UVP 3UV Lamp, Analytikjena) ubicada a $20 \mathrm{~cm}$ de altura. La irradiación se realizó por 9 minutos, tomando muestras cada minuto, lo cual equivale a dosis de radiación entre 0,22 y $1,98 \mathrm{KJ} /$ $\mathrm{m}^{2}$. Se extrapoló este dato a la exposición solar en condiciones de campo, teniendo en cuenta la radiación solar de la región, de acuerdo con el atlas de radiación solar de Colombia (www. upme.gov.co), encontrando que 10 minutos de radiación con la lámpara UV-B equivaldrá aproximadamente a 24 horas de exposición solar.

Las muestras irradiadas fueron evaluadas en un bioensayo contra larvas de $D$. saccharalis siguiendo la metodología descrita anteriormente para la determinación de concentraciones letales. La germinación de los conidios de B. bassiana Bv062, se evaluó siguiendo la metodología descrita por Grijalba et al. (2018) con modificaciones. Se tomaron $100 \mu \mathrm{L}$ de cada tratamiento y se inocularon en cajas de Petri con medio Agar agua con $0,1 \%$ de extracto de malta y $0,0005 \%$ de Benomil y se incubaron a $25 \pm 2^{\circ} \mathrm{C}$ durante 72 horas. Cada 24 horas, se cortó un cuadrado de agar de aproximadamente $1 \mathrm{~cm}^{2}$ y se adicionó una gota de azul de lactofenol. Posteriormente se cuantificó el número de conidios germinados y no germinados mediante observación al microscopio de 10 campos ópticos, por triplicado para cada unidad experimental. Se consideró como conidio germinado cuando éste presentaba un tubo germinal con longitud igual o superior al diámetro del conidio. Los tratamientos control correspondieron a conidios formulados y sin formular que no fueron expuestos a radiación.

Persistencia del bioplaguicida en la superficie foliar de plantas de caña de azúcar bajo condiciones de campo

Para la determinación de la persistencia se empleó la dosis del CE seleccionada en el ensayo mencionado anteriormente. 100 plantas de caña de la variedad CC 93-7711 de dos meses y medio de edad fueron asperjadas con una fumigadora manual RoyalCondor ${ }^{\circledR}$ Clásica de 20 L. Una hora después de la aplicación y cada semana durante cuatro semanas se seleccionaron tres plantas al azar. Sobre las hojas que habían sido aplicadas previamente con el bioplaguicida, se evaluaron dos variables:

- Recuento de conidios: se tomaron dos fragmentos de hojas de 2,25 $\mathrm{mm}^{2}$ de cada planta, uno de la hoja externa y otro de la hoja cercana a la yagua, los cuales fueron lavados con $500 \mu \mathrm{L}$ de Tween 80 al 0,1\% agitando a $200 \mathrm{rpm}$ durante dos horas. Posteriormente, en la suspensión recuperada se realizó el recuento de los conidios correspondientes a la morfología de
B. bassiana, utilizando la cámara de Neubauer.

- Actividad insecticida: de cada planta aplicada se seleccionó una hoja completa de aproximadamente $10 \mathrm{~cm}$ de largo y se colocaron en cajas de Petri plásticas que luego fueron infestadas con 15 larvas neonatas de D. saccharalis. Pasadas 24 horas, las larvas se individualizaron en recipientes plásticos de media onza que contenían un grano de maíz tierno como sustrato de alimentación. Se contó con un testigo absoluto en el cual las larvas no fueron sometidas a ningún tratamiento. Se utilizó un diseño experimental completamente al azar con tres repeticiones por tratamiento. La mortalidad de las larvas fue evaluada cada dos días hasta que todos los individuos murieron o pasaron al estado de pupa. La eficacia fue calculada con la siguiente fórmula (Zar 1999):

Eficacia $(\%)=\frac{\begin{array}{c}\% \text { de mortalidad en el tratamiento } \\ -\% \text { mortalidad en el control }\end{array}}{100-\% \text { de mortalidad en el control }} \times 100$

\section{Análisis estadístico}

Para los diferentes experimentos, la normalidad de los datos se verificó mediante la prueba de Shapiro-Wilk (95\%) y la homocedasticidad se determinó mediante una prueba de Barlett (95\%); cumplidos estos principios se realizó un análisis de varianza (ANOVA) con una confianza del 95\% $\mathrm{y}$ las diferencias entre tratamientos se calcularon mediante una prueba de Tukey. Los resultados de mortalidad del ensayo de dosis bajo condiciones semicontroladas de campo, se compararon mediante una prueba de Kruskal - Wallis, por no presentar varianzas homogéneas. Todos los análisis se realizaron utilizando el programa estadístico Statistix (8.1).

\section{RESULTADOS}

\section{Determinación de las concentraciones letales del bioplaguicida sobre larvas de $D$. saccharalis bajo condiciones de laboratorio}

El desarrollo de la infección se hizo evidente a partir del tercer día post-inoculación con reducción de la movilidad de las larvas, seguido de un cambio de coloración, desarrollo de rigidez y posterior muerte con esporulación de color blanco. Los resultados de las tres réplicas fueron promediados para el análisis y la determinación de concentraciones letales, resultados que se presentan en la Tabla 1. El análisis Probit presentó un valor de $p$ superior a 0,05 , lo cual permite concluir que hay una correlación lineal entre la concentración del hongo y la mortalidad de las larvas. La concentración letal media fue de 
$9,27 \times 10^{5}$ mientras que la noventa fue de $1,44 \times 10^{7}$ conidios/mL (Tabla 1).

Evaluación de dosis del bioplaguicida bajo condiciones semicontroladas de campo

Las tres dosis del bioplaguicida evaluadas sobre plantas de caña, presentaron una eficacia superior al $80 \%$ sin presentarse diferencias estadísticas entre las tres $(\mathrm{F}=2,03, \mathrm{gl}=8, \mathrm{p}=0,2118)$. La dosis alta $\left(6,4 \times 10^{12}\right.$ conidios/ha) alcanzó el $100 \%$ de eficacia, la dosis media $\left(3,2 \times 10^{12}\right.$ conidios/ha) alcanzó un $83,3 \pm 15,2 \%$ y la dosis baja $\left(1,6 \times 10^{12}\right.$ conidios/ha) un $96,6 \pm 5,2 \%$ de eficacia.

En cuanto al cubrimiento de las hojas con los conidios del hongo a las diferentes dosis evaluadas, se detectaron diferencias estadísticas $(\mathrm{F}=19,57, \mathrm{gl}=51, \mathrm{p}<0,001)$ entre la cantidad de conidios recuperados en las plantas aplicadas con la dosis media y alta $\left(3,8 \times 10^{5}\right.$ conidios $/ \mathrm{cm}^{2}$ y $4,6 \times 10^{5}$ conidios $/ \mathrm{cm}^{2}$, respectivamente), en comparación con las aplicadas con la dosis baja que presentó $1,2 \times 10^{5}$ conidios $/ \mathrm{cm}^{2}$ (Fig. 1). Asimismo, no se observaron diferencias significativas entre los recuentos de conidios de las dos hojas ubicadas en diferentes posiciones de la planta (hoja exterior y hoja cercana a la yagua). La viabilidad de los conidios determinada mediante recuento de UFC fue aproximadamente 10 veces menor que el valor obtenido en el recuento de conidios, en las tres dosis evaluadas (Fig. 1).

Estabilidad del bioplaguicida frente a la radiación ultravioleta tipo $B$ en condiciones de laboratorio

El efecto de la radiación ultravioleta UV-B sobre la germinación de los conidios formulados y sin formular se presenta en la Fig. 2. A medida que el tiempo de irradiación aumentó, se presentó una reducción significativa de la germinación en los dos tratamientos. Cuando la germinación fue evaluada después de 24 horas de incubación de las cajas de Petri (Fig. 2a), el comportamiento observado para los conidios formulados y sin formular fue similar. La germinación de los dos tratamientos se redujo significativamente $(\mathrm{F}=209$, $\mathrm{gl}=119, \mathrm{p}<0,0001)$ a partir de los tres minutos de irradiación (aproximadamente $45 \%$ de reducción), y a partir de allí continuó disminuyendo significativa y progresivamente a medida que aumentó el tiempo de irradiación llegando a valores finales inferiores al 10\%. La incubación de las cajas de Petri con los conidios irradiados continuó, y la germinación fue nuevamente evaluada después de 48 horas (Fig. 2b) y 72

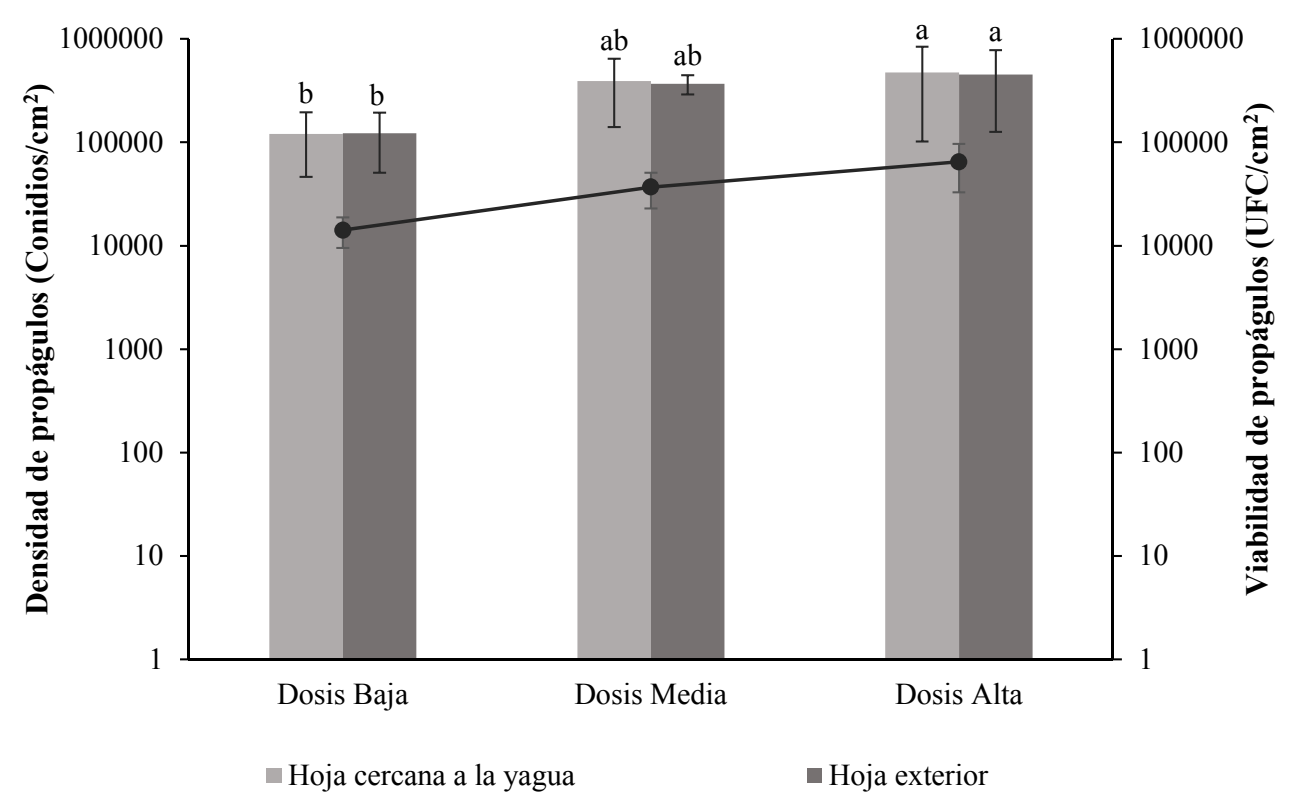

Fig. 1. Densidad de conidios en las hojas de plantas de caña de azúcar aplicadas con tres dosis del bioplaguicida CE. Dosis alta: $6,4 \times 10^{12}$ conidios/ha, dosis media: $3,2 \times 10^{12}$ conidios/ha, dosis baja: 1,4 x $10^{12}$ conidios/ha. Tratamientos con letras diferentes presentan diferencias significativas según comparación de medias de Tukey (95\%). Las barras de error representan la desviación estándar de la media.

Fig. 1. Conidia density on sugarcane leaves applied with three doses of the EC biopesticide. High dose: $6.4 \times 10^{12}$ conidia/ha, Medium dose: $3.2 \times 10^{12}$ conidia/ha, Low dose: $1.4 \times 10^{12}$ conidia/ ha. Treatments with different letters are significantly different according to Tukey's mean comparison $(95 \%)$. Error bars represent the standard deviation of the mean. 

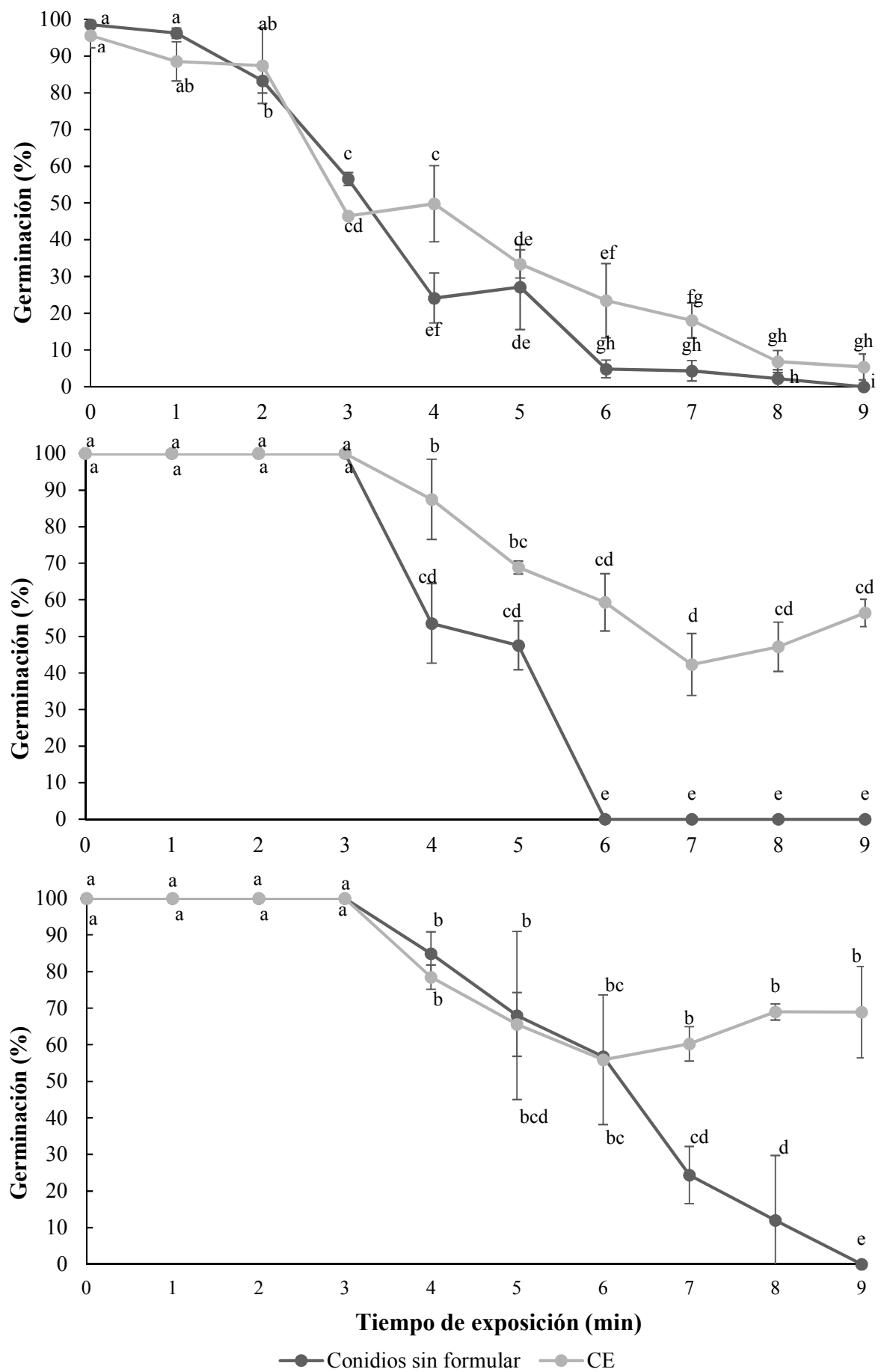

Fig. 2. Germinación de los conidios formulados (CE) y sin formular irradiados directamente con luz UV-B (302 nm) e incubados por: a) 24 horas. b) 48 horas. c) 72 horas. Tratamientos con letras diferentes son significativamente diferentes según análisis de varianza ANOVA (95\%). Las barras de error corresponden a la desviación estándar de la media.

Fig. 2. Germination of formulated conidia (EC) and non-formulated conidia directly irradiated with UV-B light (302 nm) and incubated for: a) $24 \mathrm{~h}$. B) $48 \mathrm{~h}$. C) $72 \mathrm{~h}$. Treatments with different letters are significantly different according to Tukey's mean comparison (95\%). Error bars represent the standard deviation of the mean. 
horas (Fig. 2c). A las 48 horas de incubación, la germinación de los dos tratamientos se mantuvo estable hasta los tres minutos de irradiación. Los conidios sin formular perdieron completamente su capacidad de germinación después de seis minutos, mientras que el CE no presentó cambios significativos en la germinación después de los cinco minutos de irradiación manteniendo valores entre el 42 y $68 \%$, y siendo significativamente mayores en comparación con los conidios sin formular ( $\mathrm{F}=10,6, \mathrm{gl}=119, \mathrm{p}=0,0001)$. Por último, al igual que se observó a las 48 horas de incubación, a las 72 horas la germinación de ninguno de los dos tratamientos fue significativamente afectada hasta los tres minutos de irradiación (Fig. 2c). La germinación de los conidios sin formular se redujo significativa $(\mathrm{F}=10,4, \mathrm{gl}=119, \mathrm{p}=0,0001)$ y progresivamente desde los cuatro minutos de exposición, no detectándose ningún conidio germinado a los nueve minutos de irradiación. Por el contrario, el CE presentó una reducción significativa de la germinación a los cuatro minutos $(78 \%)$, sin cambios significativos en los tiempos posteriores de evaluación (germinación final del 68,9\%). Adicionalmente, a partir de los siete minutos de irradiación los conidios sin formular presentaron valores significativamente menores $(p<0,05)$ que los obtenidos para el CE.

La actividad insecticida de los tratamientos no expuestos a la luz UV-B sobre las larvas de $D$. saccharalis fue en promedio del $96,9 \%$ y se redujo significativamente a medida que aumentó el tiempo de irradiación $(\mathrm{F}=27,2, \mathrm{gl}=119, \mathrm{p}=0,0001)$ (Fig. 3). En el caso de los conidios sin formular, a partir de los cinco minutos se evidenció una pérdida significativa de la eficacia, la cual continuó disminuyendo hasta llegar a un 20\% después de nueve minutos de irradiación. En contraste, el CE mantuvo su eficacia sin reducción hasta los siete minutos de irradiación, y posteriormente presentó una disminución de su eficacia, llegando a un $50 \%$ después de nueve minutos de irradiación.

Persistencia del bioplaguicida en la superficie foliar de plantas de caña de azúcar bajo condiciones de campo

Inmediatamente después de la aplicación del $\mathrm{CE}$, la cantidad de conidios depositados en las hojas de las plantas de caña estuvo alrededor de $1,8 \times 10^{5}$ conidios $/ \mathrm{cm}^{2}$ y se mantuvo sin una disminución significativa hasta una semana después de la aplicación (Fig. 4). Sin embargo, a partir de la segunda semana después de la aplicación, dicha concentración disminuyó significativamente hasta llegar a valores promedio de $1,1 \times 10^{4}$ conidios $/ \mathrm{cm}^{2}$, cuatro semanas después de la aplicación (Fig. 4). No se observaron diferencias significativas entre los recuentos de conidios de las dos hojas ubicadas en diferentes

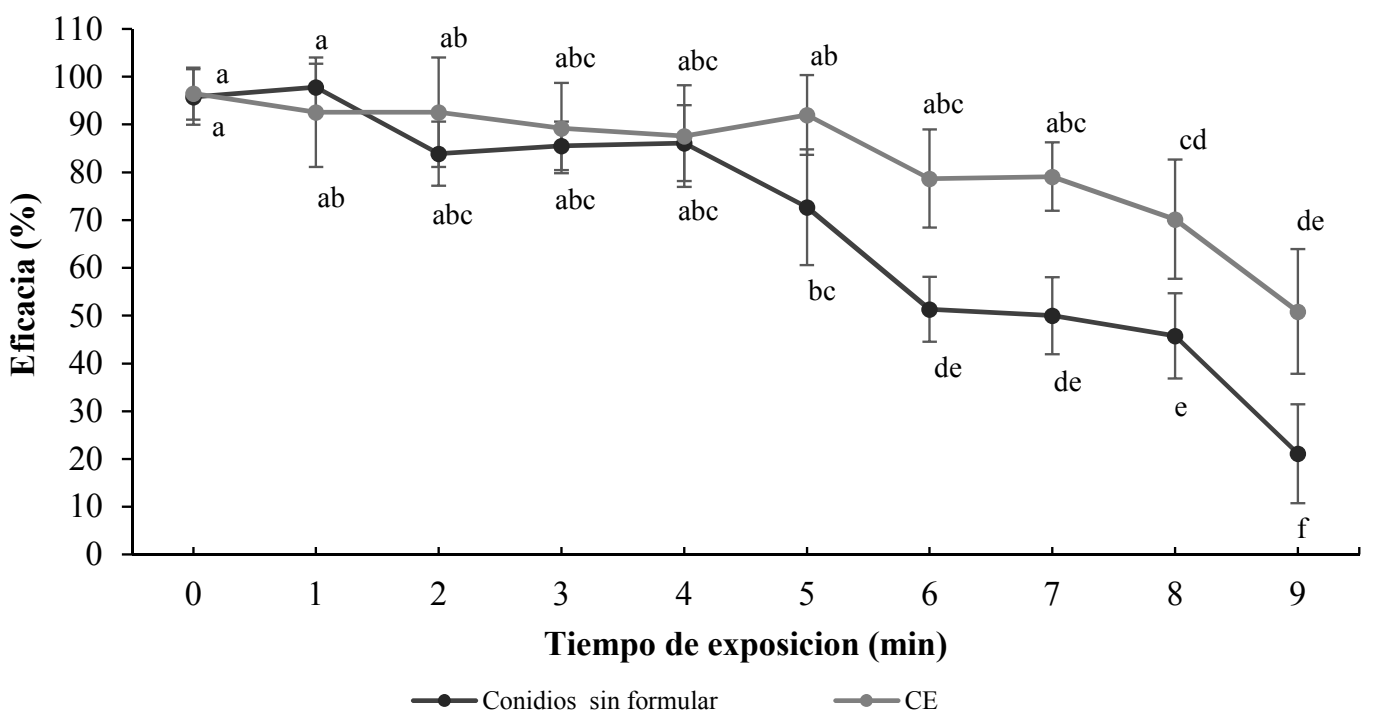

Fig. 3. Eficacia del bioplaguicida CE expuesto a radiación UV-B $(302 \mathrm{~nm})$, sobre larvas de segundo ínstar de $D$. saccharalis. Tratamientos con letras diferentes son significativamente diferentes según análisis de varianza ANOVA (95\%). Las barras de error corresponden a la desviación estándar de la media.

Fig. 3. Efficacy of the biopesticide EC exposed to UVB radiation $(302 \mathrm{~nm})$, against second instar larvae of $D$. saccharalis. Treatments with different letters are significantly different according to Tukey's mean comparison $(95 \%)$. Error bars represent the standard deviation of the mean. 


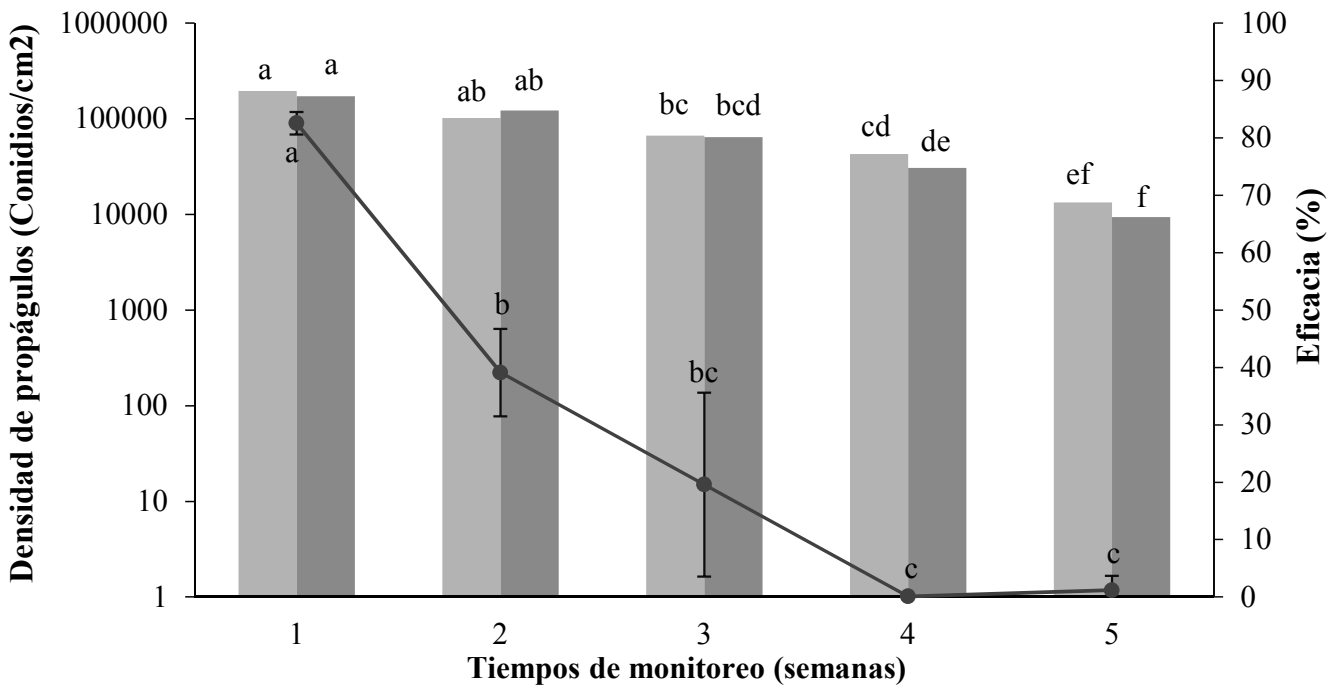

$\square$ Hoja cercana a la yagua $\quad$ Hoja exterior

Fig. 4. Persistencia de los conidios en las hojas de plantas de caña de azúcar aplicadas con el bioplaguicida CE bajo condiciones de campo. Densidad de conidios (barras) y Eficacia (Línea negra). Tratamientos con letras diferentes son significativamente diferentes según comparación de medias de Tukey ( $95 \%$ ).

Fig. 4. Persistence of conidia on sugarcane leaves applied with the EC biopesticide under field condition. Conidia density (bars) and Efficacy (black line). Treatments with different letters are significantly different according to Tukey's mean comparison $(95 \%)$. Error bars represent the standard deviation of the mean.

posiciones de la planta (hoja exterior y hoja cercana a la yagua) (Fig. 4).

De igual manera, la actividad insecticida de los conidios en las hojas de caña aplicadas se redujo del $82 \%$ al $39 \%$ desde la primera semana después de la aplicación y fue indetectable después de tres semanas (Fig. 4).

\section{DISCUSIÓN}

En un trabajo previo (Garcia et al., 2018), se determinó que el aislamiento colombiano de B. bassiana Bv062 es capaz de infectar huevos y larvas de $D$. saccharalis y otras especies de este complejo del barrenador que afectan a la caña de azúcar en Colombia. En dicho trabajo, los conidios sin formular presentaron una eficacia del $90 \%$ cuando se inoculó una concentración de $2 \times 10^{7}$ conidios/mL sobre larvas de $D$. saccharalis en condiciones controladas (García et al., 2018), resultados que motivaron el desarrollo de un prototipo de bioplaguicida formulado como un concentrado emulsionable. En la presente investigación, se evaluó el desempeño de dicho producto en laboratorio y en plantas de caña, iniciando con la determinación de las concentraciones letales, las cuales no difirieron de aquellas determinadas previamente por García et al. (2018), para los conidios sin formular de este mismo aislamiento producidos en medio en agar PDA $\left(\mathrm{CL}_{50} 9,04 \times 10^{5}\right.$ conidios $/ \mathrm{mL}$ y $\mathrm{CL}_{90} 2,07 \times 10^{7}$ conidios $/ \mathrm{mL}$ ). Estos resultados sugieren que los procesos de fermentación y formulación utilizados para la producción del CE no afectaron la actividad insecticida del hongo. La actividad insecticida de los microrganismos entomopatógenos es afectada por las condiciones utilizadas para su producción, como por ejemplo la composición del medio de cultivo, las fuentes de carbono y nitrógeno, el $\mathrm{pH}$, la actividad de agua y la temperatura entre otros (Díaz et al., 2019; Mejía et al., 2020). En un trabajo previo, la composición del medio de cultivo y de la relación C:N del mismo, demostraron ser factores determinantes para el rendimiento ecofisiológico del aislamiento Bv062, cuyos conidios producidos en un sustrato semisólido a base de papa fueron más virulentos y estables en almacenamiento que aquellos producidos en el medio convencional a base de arroz (Mejía et al., 2020).

$\mathrm{La} \mathrm{CL}_{90}$ del CE determinada en laboratorio fue usada como base para definir tres dosis de aplicación que fueron evaluadas sobre plantas de caña. Las tres dosis evaluadas resultaron 
en eficacias superiores al $80 \%$, sugiriendo alta virulencia de los propágulos fúngicos y una adecuada cobertura o distribución de los conidios en la superficie de las hojas y la yagua, la cual fue ligeramente superior en las dosis alta y media. Con base en estos resultados, se seleccionó la dosis baja del bioplaguicida $\left(1,4 \times 10^{12}\right.$ conidios/ ha) como la dosis mínima efectiva para asegurar la factibilidad económica del producto. La dosis recomendada para productos biológicos como los desarrollados a partir de hongos, es un factor crítico para su comercialización, ya que ésta debe asegurar un control eficiente de la plaga a un precio competitivo en relación con otros productos disponibles en el mercado (Ravensberg, 2011). La dosis seleccionada en el presente trabajo para el formulado a base de B. bassiana $\left(1,4 \times 10^{12}\right.$ conidios/ha) es similar a la dosis reportada para otras formulaciones a base de $B$. bassiana evaluadas para el control de $D$. saccharalis, en donde una aplicación de $10^{12}$ conidios/ha de una formulación granular a base de B. bassiana logró disminuir los niveles poblacionales del insecto en un $67 \%$, así como los entrenudos barrenados en un 63\% (Estrada et al., 1997). Estos resultados sugieren la necesidad de continuar determinando los factores claves en la aplicación del bioplaguicida en condiciones de campo.

Respecto a la distribución del hongo en la superficie foliar, el resultado encontrado en el presente trabajo permitió concluir que las condiciones de aplicación utilizadas favorecieron una distribución homogénea del producto, obteniéndose densidades de conidios iguales en las hojas externas y en las hojas cercanas a la yagua. Una dispersión homogénea del producto en las plantas de caña es importante, ya que ésta determina la probabilidad de que las larvas se infecten con los conidios del hongo, considerando el modo de acción tópico del hongo y que las larvas neonatas de $D$. saccharalis recorren las hojas varias veces antes de ubicarse en la zona del nudo (entre el tallo y la yagua) (Gómez y Miranda, 2009; Cardona y Soto, 2015). Sin embargo, es importante resaltar que cada parte de la planta genera un microclima distinto que puede afectar la actividad biológica del hongo, debido a diferencias en las condiciones (Jaronski, 2010). El tipo y calidad de la formulación también desempeñan un papel fundamental en la cobertura de las plantas y la dispersión del hongo entomopatógeno en el campo. En el presente trabajo, los conidios fueron formulados como un $\mathrm{CE}$, cuya composición incluye un vehículo oleoso y tensioactivos que permiten la emulsificación del producto al momento de su reconstitución en agua. La formación de una emulsión estable y homogénea, asegura una distribución uniforme del ingrediente activo (conidios) en el tanque del equipo aspersor y una mejor cobertura de la superficie foliar (Batta, 2016). El carácter oleoso de la formulación CE evaluada en este estudio pudo haber sido determinante en la dispersión y eficacia del producto, así como lo reportaron Guinossi et al. (2012), quienes concluyeron que la aplicación de los conidios de B. bassiana suspendidos en aceite mejoran su dispersión en cultivos de soya.

El recuento de UFC a partir de las muestras recuperadas de las laminillas fue hasta diez veces menor que los resultados obtenidos mediante recuento en cámara de Neubauer. Esta diferencia ya ha sido reportada previamente, y posiblemente se encuentra relacionada con las diferencias en los principios y limitaciones de los dos métodos, ya que el recuento de conidios en el microscopio no permite diferenciar los conidios viables de los no viables y en el recuento en placa, no siempre un conidio da origen a una colonia y una UFC puede formarse a partir de un conglomerado de propágulos (Oliveira et al., 2015). En ese sentido, ambos métodos deben ser afinados con el fin de obtener una correlación entre la presencia del hongo en las hojas y su viabilidad.

Otro de los grandes desafíos que enfrentan los bioplaguicidas a base de hongos entomopatógenos, es garantizar la protección de los propágulos fúngicos frente a factores de estrés abiótico que afectan su efectividad y persistencia en campo. La radiación ultravioleta proveniente de la luz solar es considerada como uno de los factores medioambientales más perjudiciales para la viabilidad de los conidios cuando son utilizados en campo (Fernandes et al., 2015). En este trabajo se determinó el efecto negativo de la radiación UV sobre la germinación de los conidios formulados y sin formular, efecto que fue mayor a medida que aumentó el tiempo de irradiación. Esta disminución fue más evidente para los conidios sin formular, lo que indica que la formulación confiere protección a los conidios, posiblemente debido a la absorción de luz UV por el vehículo oleoso y por los filtros ultravioleta incluidos en ésta. Cabe destacar que se obtuvieron resultados de inactivación diferentes en los tres tiempos de lectura de la germinación de los tratamientos (24, 48, y 72 horas), con un aumento de la germinación a medida que aumentó el tiempo de lectura (Fig. 3). Este comportamiento indica que los conidios sobrevivientes requirieron un periodo de tiempo mayor a 24 horas para recuperarse de los efectos causados por la radiación ultravioleta, posiblemente mediante la activación de mecanismos reparación. Esto fue evidenciado por Fernandes et al. (2007), quien 
describió que, el retraso de la germinación de aislamientos de $B$. bassiana expuestos a radiación ultravioleta UV-B pudo ser debido a la inducción de sistemas de reparación, como duplicación del material genético dañado y segregación de cromosomas fracturados. Este resultado fue corroborado por los resultados de eficacia, que demostraron que los conidios no perdieron completamente su actividad insecticida a pesar de mostrar valores bajos de germinación.

El ensayo de persistencia en campo demostró que la actividad insecticida se redujo en aproximadamente un $50 \%$ después de la primera semana de la aplicación sobre plantas de caña, y continuó disminuyendo a medida que avanzó el tiempo de exposicion a las condiciones ambientales. La pérdida de actividad insecticida determinada en el presente trabajo podría ser resultado de las drásticas condiciones climáticas que se presentaron durante el desarrollo de los experimentos, caracterizadas por fuertes lluvias y alta radiación solar. Es importante evaluar otros factores como la luz solar, la lluvia, la temperatura, la humedad, la química de la superficie de la hoja, la microbiota del filoplano que también pueden afectar la persistencia y eficacia de los micoinsecticidas aplicados en plantas, como lo describió Jaronski (2010). La precipitación es un factor determinante para la persistencia de los conidios de hongos entomopatógenos en la superficie foliar, pudiendo disminuir entre un 28 y $61 \%$ el recuento de conidios en las hojas (Inglis et al., 1995; Inglis et al., 2000; Tamez-Guerra et al., 2000). Esta limitante se puede mitigar incluyendo en la formulación o mezclando con esta en el momento de la reconstitución, adjuvantes que aumenten la adherencia del producto. A pesar de que las formulaciones tipo $\mathrm{CE}$ favorecen la adherencia de los conidios por su naturaleza oleosa, existen también compuestos poliméricos que podrían complementar esta función, como el látex y el alcohol polivinilico (Feng et al., 2018). En ese sentido, es importante notar que los recuentos de conidios en las hojas no presentaron una disminución drástica durante la semana después de la aplicación del bioplaguicida, mientras que la eficacia se redujo significativamente sugiriendo que los conidios persisitieron en las hojas pero perdieron su actividad insecticida o su viabilidad. Esto pudo deberse al efecto de la radiación ultravioleta del sol, dado que aunque la formulación confirió cierto nivel de fotoprotección, ésta no fue del $100 \%$. A pesar de la reducción en la eficacia del CE después de su aplicación en plantas de caña, fue posible evidenciar actividad insecticida hasta después de dos semanas, lo que contrasta con los resultados de Estrada y Guelmes (2004), quienes evaluaron la persistencia de otro aislamiento de $B$. bassiana en hojas de caña de azúcar en condiciones experimentales de campo. Estos autores encontraron que los propágulos del hongo permanecieron viables sobre las hojas sólo durante 48 horas después de la aplicación a plantas que estaban expuestas a condiciones de luz solar. En este sentido, la formulación del hongo empleada en esta investigación demostró beneficios en la protección de los conidios en comparación con la aplicación de conidios sin formular. Sin embargo, es importante optimizar el prototipo de bioplaguicida a base de B. bassiana Bv062, con el fin de mejorar su desempeño, junto con la evaluación de otras estrategias de aplicación que favorezcan la dispersión y establecimiento del hongo en el ecosistema de los cultivos de caña. Asimismo, se hace necesario continuar con los ensayos en condiciones de campo, con el fin de determinar las recomendaciones de aplicación del bioplaguicida y validar su uso en diferentes condiciones agroclimáticas.

En conclusión, el prototipo de bioplaguicida CE a base de conidios de Bv062 demostró un potencial interesante para el control de $D$. saccharalis, sugiriendo la necesidad de continuar con su desarrollo y optimización, que junto con la validación de su eficacia en campo, dará viabilidad a su posterior escalamiento, registro y comercialización.

\section{AGRADECIMIENTOS}

A Richard Sánchez, Javier Jiménez de Agrosavia (Sede Cimpa) por su apoyo en la toma de muestras, mantenimiento de las plantas y colaboración en los lotes en campo. Este estudio fue financiado por el Ministerio de Agricultura y Desarrollo Rural de Colombia con fondos gubernamentales asignados a la Corporación Colombiana de Investigación Agropecuaria (AGROSAVIA) (Proyecto: "Recomendaciones para el manejo de Diatraea spp. mediante métodos biológicos y etológicos"). El apoyo a la investigación fue proporcionado por fondos gubernamentales asignados a AGROSAVIA.

\section{LITERATURA CITADA}

Aya, V.M., J. Montoya, C. Echeverri, and J.P. Michaud, G. Vargas. 2019. Partial host resistance to two parasitoids (Diptera: Tachinidae) helps explain a regional outbreak of novel Diatraea spp. stem borers (Lepidoptera: Crambidae) in Colombia sugarcane. Biological Control 129:18-23. doi:10.1016/j.jcat.2003.10.016. 
Batta, Y.A. 2016. Invert emulsion: Method of preparation and application as proper formulation of entomopathogenic fungi. MethodsX 3:119-127. doi:0.1016/j. mex.2016.02.001.

Cardona, L. y A. Soto. 2015. Susceptibilidad de Diatraea saccharalis (F) (Lepidoptera:Crambidae) a diferentes hongos entomopatógenos en caña panelera. Boletín científico Museo de Historia Natural Universidad de Caldas 19(2):95-103.

Díaz, A., M. Gómez-Álvarez, E. Grijalba, A. Santos, F.M. Cruz, D.M. León y E.A. Alarcón, A.M. Cotes. 2019. Desarrollo y escalamiento de bioplaguicidas. p. 628-682. En: A.M. Cotes (ed). Control biológico de fitopatógenos, insectos y ácaros. Volumen 2. Aplicaciones y perspectivas. AGROSAVIA Editorial, Mosquera, Cundinamarca, Colombia.

Estrada, M. y J. Guelmes. 2004. Persistencia de Beauveria bassiana (Bálsamo) Vuillemin en las hojas de caña de azúcar (Saccharum sp. Híbrido). Fitosanidad 8(4):53-56.

Estrada, M.E., M. Romero y M. Snowball. M. 1997. Aplicación de Beauveria bassiana en la Lucha biológica contra Diatraea saccharalis. Revista Caña de azúcar 15(1):39-43

Feng, J., Q. Zhang, Q. Liu, Z. Zhu, and D.J. McClements, S.M. Jafari. 2018. Application of Nanoemulsions in Formulation of Pesticides. Elsevier Inc.

Fernandes, É.K.K., D.E.N. Rangel, and G.U.L. Braga, and D.W. Roberts. 2015. Tolerance of entomopathogenic fungi to ultraviolet radiation: a review on screening of strains and their formulation. Current Genetics. 61(3):427-440. doi: 10.1007/s00294-015-0492-z

Fernandes, É.K.K., D.E.N. Rangel, Á.M.L. Moraes, V.R.E.P. Bittencourt, and D.W. Roberts. 2007. Variability in tolerance to UV-B radiation among Beauveria spp. isolates. Journal of Invertebrate Pathology. 96(3):237-243. doi: 10.1016/j.jip.2007.05.007.

García, J.L., P. Sotelo, D.M. Monroy, G. Barrera, J. Gómez-Valderrama, C. Espinel, E., Barreto., and L.F. Villamizar. 2018. Identification and characterization of a Beauveria bassiana (Bals.) Vuill. isolate having a high potential for the control of the Diatraea sp. sugarcane stem borer. Biotecnología Aplicada 35(1):12011207.

Gómez, E. y J. Miranda. 2009. Manejo agronómico de la caña panelera con énfasis en el control biológico. 32 p. Fondo Nacional de la Panela. Roffaprint Editores. Bogotá, Colombia.
Grijalba, E.P, C. Espinel, P.E. Cuartas, M.L. Chaparro, and L.F. Villamizar. 2018. Metarhizium rileyi biopesticide to control Spodoptera frugiperda: Stability and insecticidal activity under glasshouse conditions. Fungal Biology. 122:1069-1076. doi: 10.1016/j.funbio.2018.08.010.

Guinossi, H.D.M., F. Moscardi, M.C.N. Oliveira, and D.R. Sosa-Gómez. 2012. Spatial dispersal of Metarhizium anisopliae and Beauveria bassiana in soybean fields. Tropical Plant Pathology.37(1):44-49. doi:10.1590/S198256762012000100005.

Inglis, D., T.J. Ivie, G.M. Duke, and M.S. Goettel. 2000. Influence of Rain and Conidial Formulation on Persistence of Beauveria bassiana on Potato Leaves and Colorado Potato Beetle Larvae. Biological Control. 18(1):55-64. doi: 10.1006/bcon.1999.0806.

Inglis, D., D.L. Johnson, and M.S. Goettel. 1995. Effects of Simulated Rain on the Persistence of Beauveria bassiana Conidia on Leaves of Alfalfa and Wheat. Biocontrol Science and Technology. 5(3):365-370. doi: 10.1080/09583159550039819.

Inglis, G.D., D. Jhonson, and M. Goettel. 1997. Field and Laboratory evaluation of two conidial batches of Beauveria bassiana ( Balsamo) Vuillemin against Grasshoppers. The Canadian Entomologist. 129:171-1186

Jaronski, S.T. 2010. Ecological factors in the inundative use of fungal entomopathogens. BioControl. 55:159-185. doi:10.1007/978-90481-3966-8_12.

Lastra, B. y L.A. Gómez . 2006. La cría de Diatraea saccharalis (F.) para la producción masiva de sus enemigos naturales. Serie Técnica Ceñicaña. 36(3):30

Legaspi, J.C., T.J. Poprawski, and A.B.C. Legaspi. 2000. Laboratory and Field Evaluation of Beauveria bassiana Against Sugarcane Stalkborers (Lepidoptera: Pyralidae) in the Lower Rio Grande Valley of Texas. Journal of Economic Entomology. 93(1):54-59.

Mascarin G., and S. Jaronski. 2016. The production and uses of Beauveria bassiana as a microbial insecticide. World Journal Microbiology Biotechnology. 32 (177): 1-26.

Mejía, C., C. Espinel, M. Forero, F.A. Ramos, F.B. Pedro, and L.F. Villamizar. 2020. Improving ecological fitness of Beauveria bassiana conidia to control the sugar cane borer Diatraea saccharalis. Biocontrol Science and Technology. 30 (6):513-530. doi: 10.1080/09583157.2020.1738343. 
Oliveira, D.G.P., G. Pauli, G.M. Mascarin, and I. Delalibera. 2015. A protocol for determination of conidial viability of the fungal entomopathogens Beauveria bassiana and Metarhizium anisopliae from commercial products. Journal of Microbiological Methods. 119:44-52. doi: 10.1016/j. mimet.2015.09.021.

Ravensberg, W.J. 2011. Progress in Biological Control: A roadmap to the successful development and comercialization of microbial pest contro products for control of arthropods. p. 383. Springer. Dordrecht, The Netherlands.

Tamez-Guerra, P., M.R. McGuire, R.W. Behle, J.J. Hamm, H.R. Sumner, and B.S. Shasha. 2000. Sunlight persistence and rainfastness of spray-dried formulations of baculovirus isolated from Anagrapha falcifera (Lepidoptera: Noctuidae). Journal of Economic Entomology. 93(2):210-8
Vargas, G., L.A. Gómez, and J.P. Michaud. 2015. Sugarcane Stem Borers of the Colombian Cauca River Valley: Current Pest Status, Biology, and Control. Florida Entomologist. 98(2):728-735. doi: 10.1653/024.098.0249.

Vargas, G., L.A. Lastra, and M.A. Solís. 2013. First Record of Diatraea tabernella (Lepidoptera: Crambidae) in the Caucariver Valley of Colombia. Florida Entomologist. 96(3):11981201. doi: $10.1653 / 024.096 .0367$

Zar, J. 1999. Biostatistical Analysis. p. 663. Prentice H. New Jersey. 